ARTICLE

Received 2 Jan 2014 | Accepted 14 Jul 2014 | Published 26 Aug 2014

DOI: $10.1038 /$ ncomms5686

OPEN

\title{
Unconventionally secreted effectors of two filamentous pathogens target plant salicylate biosynthesis
}

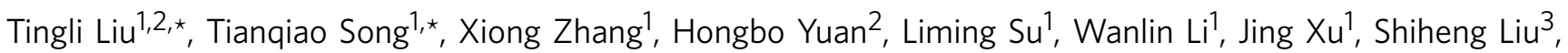
Linlin Chen ${ }^{1}$, Tianzi Chen², Meixiang Zhang ${ }^{1}$, Lichuan Gu ${ }^{3}$, Baolong Zhang ${ }^{2} \&$ Daolong Dou ${ }^{1}$

Plant diseases caused by fungi and oomycetes pose an increasing threat to food security and ecosystem health worldwide. These filamentous pathogens, while taxonomically distinct, modulate host defense responses by secreting effectors, which are typically identified based on the presence of signal peptides. Here we show that Phytophthora sojae and Verticillium dahliae secrete isochorismatases (Pslsc1 and Vdlsc1, respectively) that are required for full pathogenesis. Pslsc1 and Vdlsc1 can suppress salicylate-mediated innate immunity in planta and hydrolyse isochorismate in vitro. A conserved triad of catalytic residues is essential for both functions. Thus, the two proteins are isochorismatase effectors that disrupt the plant salicylate metabolism pathway by suppressing its precursor. Furthermore, these proteins lack signal peptides, but exhibit characteristics that lead to unconventional secretion. Therefore, this secretion pathway is a novel mechanism for delivering effectors and might play an important role in host-pathogen interactions.

\footnotetext{
${ }^{1}$ Department of Plant Pathology, Nanjing Agricultural University, 1 Weigang Road, Nanjing 210095, China. ${ }^{2}$ Provincial Key Laboratory of Agrobiology, Jiangsu Academy of Agricultural Sciences, Nanjing 210095, China. ${ }^{3}$ State Key Laboratory of Microbial Technology, Shandong University, Jinan 250100, China.

* These authors contributed equally to this work. Correspondence and requests for materials should be addressed to D.D. (email: ddou@njau.edu.cn) or to B.Z. (email: zhbl2248@hotmail.com).
} 
$\mathrm{P}$ hytophthora sojae is an oomycete pathogen from soybean ${ }^{1}$, whereas Verticillium dahliae is a vascular wilt fungus that causes diseases in $>200$ plant species ${ }^{2}$. Although they are evolutionarily distant eukaryotes, both are filamentous pathogens that grow or infect their hosts via thread-like hyphae. These filamentous plant pathogens secrete a complex repertoire of effector proteins to manipulate host innate immunity ${ }^{3,4}$. The effectors can either contribute to pathogen virulence by overcoming plant defense (termed effector-triggered susceptibility), or be directly or indirectly recognized by host surveillance systems, leading to effector-triggered immunity ${ }^{5}$. Typically, the secreted effectors are characterized by short $\mathrm{N}$-terminal signal peptides that direct them to the secretory pathway $^{3,6}$. P. sojae produces hundreds of apoplastic effectors and putative cell-entering effectors ${ }^{1,3,6}$, whereas $V$. dahliae secretes more than 700 putative effectors, including many small cysteinerich proteins that are thought to function in the host apoplast to promote disease ${ }^{2}$. For example, the Verticillium effector Ave1 contributes to host fungal virulence in the absence of its corresponding $\mathrm{R}$ protein $(\mathrm{Ve} 1)^{7}$. Nevertheless, the virulence targets and mode of action of these effectors remain largely unknown.

Salicylate (SA) is a central signaling molecule during plant innate immune responses. It is usually induced by infection with biotrophic or hemibiotrophic pathogens, and serves as an important component of PAMP-triggered immunity (PTI), effector-triggered immunity and systemic acquired resistance ${ }^{8,9}$. Plants synthesize SA through two distinct enzymatic pathways. One is from phenylalanine via cinnamate and the other is from chorismate via isochorismate $e^{8,10}$. The second pathway is also shared by some bacteria. For example, Pseudomonas aeruginosa produces SA via two distinct enzymes: isochorismate synthase (ICS) and isochorismate pyruvate lyase $^{11}$. ICS homologues have been identified in a wide variety of plant species and genetic analyses revealed that the ICS-dependent SA biosynthetic pathway predominates under (a)biotic stresses in Arabidopsis, although IPL genes have not yet been identified in plants ${ }^{8,10}$.

Isochorismate is a precursor of both SA and many other distinct derivatives in plants, fungi and bacteria ${ }^{10}$. For example, in bacteria, isochorismate can be converted into 2,3-dihydro-2,3dihydroxybenzoate (DDHB) and pyruvate by isochorismatase (ISC, EC 3.3.2.1). Escherichia coli EntB and Vibrio cholerae VibB contain a conserved amino-terminal ISC domain and carboxy-terminal aryl carrier protein domain. They play roles in the production of enterobactin and vibriobactin, respectively ${ }^{12,13}$. Pseudomonas aeruginosa $\mathrm{PhzD}$ is required for phenazine biosynthesis; it is $46 \%$ identical to EntB, but it lacks its C-terminal domains. PhzD also catalyses the hydrolysis of isochorismate ${ }^{14}$. Isochorismatase-like hydrolases have been identified from the pathogenic protozoans Leishmania donovani, L. major and Trypanosoma cruzi ${ }^{15}$, several phytopathogenic fungi ${ }^{16}$ and the rice root nematode Hirschmanniella oryzae ${ }^{17}$. However, their functions in these pathogens remain unknown.

Here we identified two genes from P. sojae (PsIsc1) and $V$. dahliae (VdIsc1) whose putative encoded proteins share high similarity with known ISCs and exhibit ISC activity. We demonstrated that both genes were required for virulence and that they function by suppressing SA accumulation in their hosts. The genes encode unconventionally secreted proteins and PsIsc1 could mediate the translocation of the $\mathrm{C}$ terminus of the $P$. sojae effector Avrlb into host cells. The in planta expression of these genes reduced SA levels. We propose that these two unconventionally secreted effectors hydrolyse host isochorismate, thereby modulating the SA metabolism pathway and suppressing host immunity.

\section{Results}

PsIsc1 and VdIsc1 are putative ISCs. As ISC can hydrolyse isochorismate (the direct precursor of SA), we hypothesized that some phytopathogens might exploit ISCs as virulence effectors to suppress host SA biosynthesis. Therefore, we searched for P. sojae and $V$. dahliae ISCs using reciprocal BLAST searches with 40 known ISCs from the PDB database (Supplementary Table 1). Of 17 candidates, three each from $P$. sojae and $V$. dahliae were identified as putative ISCs based on HHpred searches ${ }^{18}$ (Supplementary Table 2). Of these 6 candidate genes, one from each species (PsIsc1 and VdIsc1) was upregulated during the infection stages (Supplementary Fig. 1). The predicted PsIsc1 and VdIsc1 proteins shared high primary and secondary structural similarities with the reported ISCs (Supplementary Fig. 2). Thus, they are candidate virulence proteins in these two filamentous pathogens.

PsIsc1 is a virulence factor in $P$. sojae. To determine the role of PsIsc1 in P. sojae virulence, we silenced or overexpressed the gene using polyethylene glycol-mediated protoplast transformation ${ }^{19}$. The PsIsc1 transcripts were reduced to $38 \%$ and $18 \%$ in two silenced transgenic lines (ST6 and ST22, respectively) and increased by 90 - and 376-fold in two overexpression transgenic lines (OT3 and OT20, respectively) (Fig. 1a). Western blotting confirmed that PsIsc1 was successfully expressed at the expected size in OT3 and OT20 (Fig. 1b). All transformants showed normal filamentous growth. Compared with the wild-type (WT) strain and a transgenic line (T10) with a normal PsIsc1 expression level, the two silenced lines (ST6 and ST22) showed a visible reduction in virulence on soybean seedlings, whereas the two overexpressed lines (OT3 and OT20) were more virulent than the controls (Fig. 1c,d).

To further characterize PsIsc1-mediated virulence, we measured the SA levels in soybean seedlings infected with the P. sojae transformants. PsIsc1 silencing increased the concentrations of both free SA and its glucoside (SAG) by $\sim 3$-fold. In contrast, PsIsc1 overexpression significantly decreased the concentrations of SA and SAG (Fig. 1e). The transcript levels of the SA-inducible PATHOGENESIS-RELATED 1 (PR1) gene were significantly increased or reduced accordingly at sites inoculated with the transformants compared with WT and T10 (Fig. 1f). Collectively, our results suggest that PsIscl functions as a virulence factor in $P$. sojae by suppressing host SA accumulation.

VdIscl is required for full virulence in $\boldsymbol{V}$. dahliae. To explore the role of $V d I s c 1$ in $V$. dahliae virulence, deletions were generated in $V$. dahliae strain V991 to produce two mutants: $\Delta 1-12$ (V991 $\Delta V d I s c 1-12)$ and $\Delta 1-18$ (V991 $\Delta V d I s c 1-18)$. Two mutants exhibited normal growth phenotypes (Supplementary Fig. 4). Based on pathogenicity tests in the cotton cultivar Lumian 21, the two VdIsc1 deletion mutants produced significantly reduced disease symptoms. To confirm that the mutant phenotypes resulted from the deletion of $V d I s c 1$, a single copy of the gene was introduced into $\Delta 1-12$. The resulting complemented strain, $\Delta 1-12$ VdIsc1, was fully pathogenic and induced disease at rates similar to the WT strain. We also explored the ability of PsIsc1 to complement the $\Delta 1-12$ deletion. PsIsc1 could functionally replace VdIsc1 (Fig. 2a and Supplementary Fig. 4), suggesting that the two genes play similar roles in virulence.

In cotton plants inoculated with the two deletion mutants, the free SA and SAG levels were increased by $100-150 \%$ (Fig. 2b) and there was a corresponding $\sim 2$-fold increase in the level of cotton PR1 mRNA (Fig. 2c). The concentration of SA, SAG and PR1 transcript levels after infection with the VdIsc1- and PsIsc1complemented transformants were comparable with the WT 
a

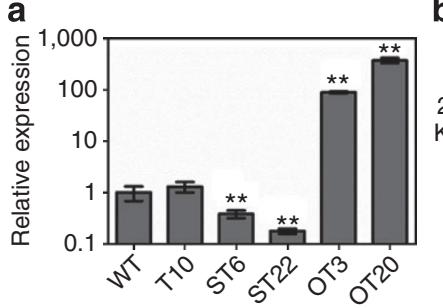

b

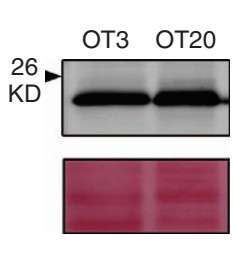

c

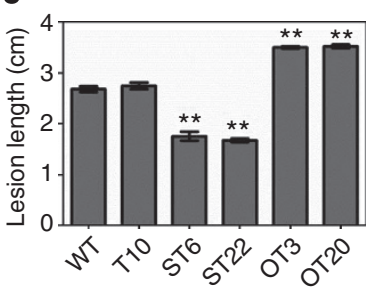

d
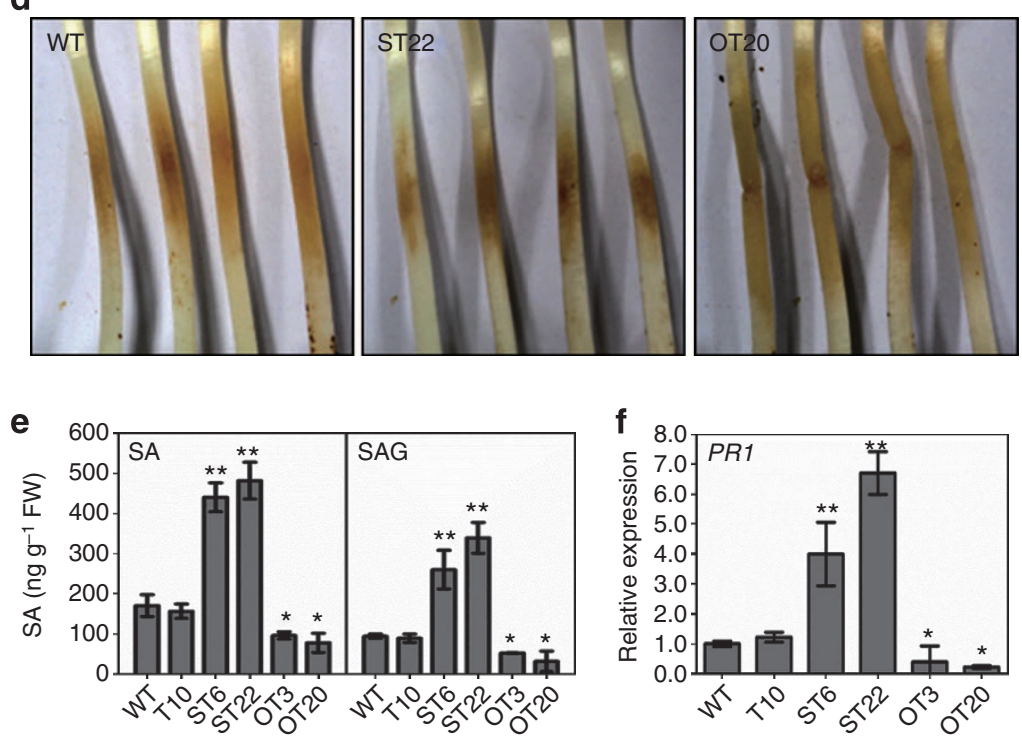

Figure 1 | Pslsc1 is a virulence factor in P. sojae. (a) Relative levels of Pslsc1 transcripts in $P$. sojae transformants measured using qRT-PCR. Relative

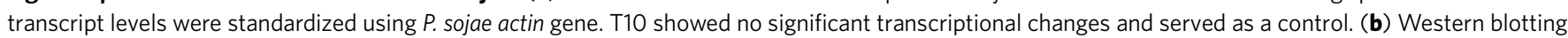
of proteins from P. sojae transformants expressing Pslsc1-His (OT3 and OT20). Anti-His antibody was used and a band at the expected size was detected (upper panel). Total proteins were stained using Ponceau S (lower panel). (c) Lesion lengths of soybean-etiolated seedling hypocotyls inoculated with 100 zoospores (36 hpi). (d) Phenotypes of soybean-etiolated seedlings inoculated with $P$. sojae transgenic lines. Typical photos taken at 36 hpi are shown.

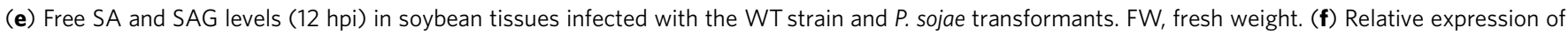
soybean PR1 in plants infected with the WT strain and transgenic lines at 12 hpi. Expression was normalized against soybean actin. In a-f, bars represent the s.e. from at least three independent replicates. Asterisks indicate significant differences ( ${ }^{\star} P<0.05 ;{ }^{\star \star} P<0.01 ;$ Dunnett's test) between each transgenic line and the WT control.

(Fig. 2b,c). Thus, both genes can act as virulence factors in $V$. dahliae by reducing host SA levels. Both PsIsc1 and VdIsc1 contain a triad of catalytic residues (Asp-Lys-Cys; D-K-C), which form the activate sites and are responsible for the features of many known ISCs ${ }^{15,20}$ (Supplementary Fig. 2). We generated substitution mutations of the three key amino acids in both genes $\left(P s I s c 1^{A 3}\right.$ and $\left.V d I s c h 1^{A 3}\right)$ and found that neither mutant could complement the $V$. dahliae $\Delta V d I s c 1$ mutant based on virulence tests (Fig. 2a), suggesting the virulence contributions of these effectors are probably linked to their enzymatic activities.

To elucidate the biological significance of the elevated SA levels during infection with the deletion mutants, we estimated the effect of SA on cotton resistance. The results revealed that pretreatment with SA reduced virulence, comparable to $\Delta 1-12$ infections (Supplementary Fig. 5). This suggests that SA enhances the resistance of cotton toward $V$. dahliae. Furthermore, we analysed the virulence contributions of VdIscl using the Arabidopsis thaliana WT and NahG transformant plants. SA levels are extremely low in Arabidopsis plants that expresses the Pseudomonas putida NahG gene, because it encodes salicylate hydroxylase, an enzyme that converts SA to catechol ${ }^{21}$. As shown in Fig. 2d, the NahG transformant was significantly more susceptible to $V$. dahliae than $A$. thaliana WT, supporting the hypothesis that SA is required for plant resistance to $V$. dahliae.
$\Delta 1-12$ or $\Delta 1-12 \mathrm{VdIsc}^{\mathrm{A} 3}$, but not $\Delta 1-12 \mathrm{VdIsc1}$, exhibited significantly reduced virulence in $A$. thaliana WT. In contrast, the NahG transformant showed comparable susceptibility to $V$. dahliae WT, $\Delta 1-12, \Delta 1-12 \mathrm{VdIsc1}$ and $\Delta 1-12 \mathrm{VdIsc}^{\mathrm{A} 3}$. Taken together, these results suggest that VdIscl suppress plant SA-mediated immunity, which could be a direct consequence of its inability to interfere with SA biosynthesis in the host plants.

PsIsc1 and VdIsc1 are unconventionally secreted proteins. To identify the site of action of VdIscl and PsIsc1 during interactions, we analysed whether they were secreted. Neither protein was predicted to contain an $\mathrm{N}$-terminal signal peptide that could direct the protein to the conventional secretory pathway (Supplementary Table 2 and Supplementary Fig. 2). However, both were predicted to be non-classically secreted based on SecretomeP 2.0 (ref. 22), with NN scores (neural network output scores) of 0.66 and 0.81 , respectively (Supplementary Table 2), which is above the threshold of 0.5 . To test the predictions experimentally, we used western blotting to detect secreted proteins in culture supernatants of the $P$. sojae transgenic line OT20 and the $V$. dahliae complemented line $\Delta 1-12 \mathrm{VdIsc1}$, which produced His-tagged PsIsc1 and haemagglutinin (HA)-tagged 

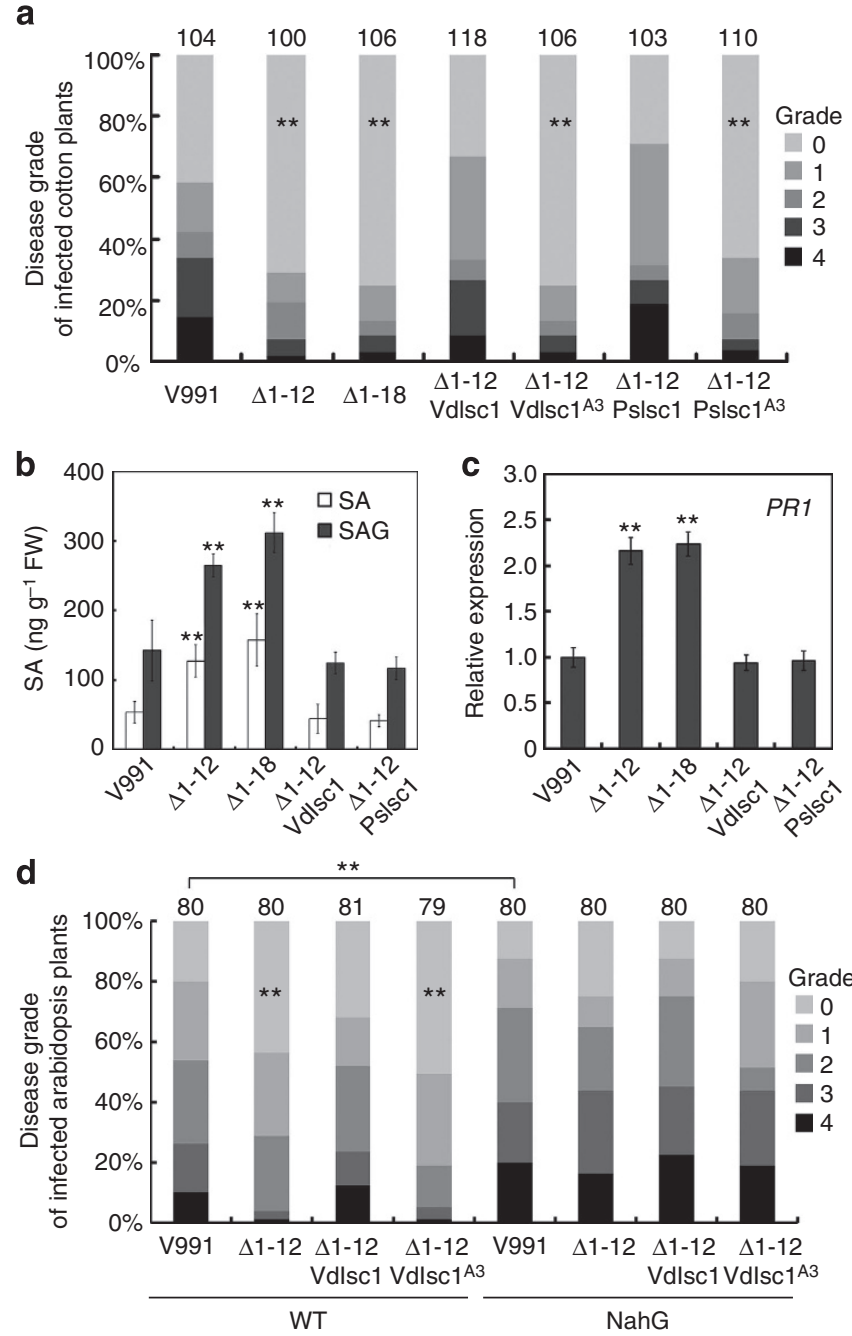

Figure 2 | Functional Vdlsc1 or Pslsc1 is required for full V. dahliae virulence. (a) Disease symptoms on cotton plants were scored at $15 \mathrm{dpi}$ with the indicated strains. The disease grade is depicted in Supplementary Fig. 3. The total number of infected plants is indicated above each column ( ${ }^{\star \star} P<0.01$; $t$-test). $\vee 991$ represents the $\mathrm{WT} V$. dahliae isolate; $\Delta 1-12$ (V991 $\Delta$ Vdlsc1-12) and $\Delta 1-18$ (V991 $\Delta$ Vdlsc1-18) represent two strains in which Vdlsc1 was deleted; $\Delta 1-12 \mathrm{Vdlsc1}, \Delta 1-12 \mathrm{Vdlsc} \mathrm{A}^{\mathrm{A} 3}, \Delta 1-12 \mathrm{Pslsc1}$ and $\Delta 1-12$ Pslsc1 $^{\mathrm{A} 3}$ represent, respectively, $\Delta 1-12$ complemented with $V d l s c 1$, with a Vdlsc1 mutant encoding three key amino acid substitutions (D26A, K100A and $\mathrm{C} 133 \mathrm{~A}$ ), with Ps/sc1 and with a Ps/sc1 mutant encoding comparable substitutions (D25A, K90A and C124A; Supplementary Fig. 2). All complementing constructs used the Aspergillus nidulans trpC promoter. (b) Free SA and SAG levels in cotton roots at $14 \mathrm{dpi}$. (c) Relative levels of cotton PR1 transcripts measured using qRT-PCR at $12 \mathrm{hpi}$. Expression was normalized against the constitutively expressed cotton gene Histone3. In $\mathbf{b}$ and $\mathbf{c}$, the data represent the mean \pm s.d.; ${ }^{\star \star} P<0.01$ (Dunnett's test) between WT and each transgenic line. (d) Disease symptoms of the Arabidopsis WT and NahG-transformed plants. The disease grades are described in the Methods. The total number of infected plants is shown above each column ${ }^{\star \star} P<0.01$; $t$-test $)$

VdIsc1, respectively. PsIsc1-His and VdIsc1-HA could be detected in culture supernatants, as well as in hyphae of the respective transformants. However, the control cytoplasmic protein, $\alpha$-actin, was only found in the hyphae of both cases (Fig. 3). Thus, PsIsc1 and VdIsc1, although they lack classical N-terminal signal peptides, are secreted proteins.
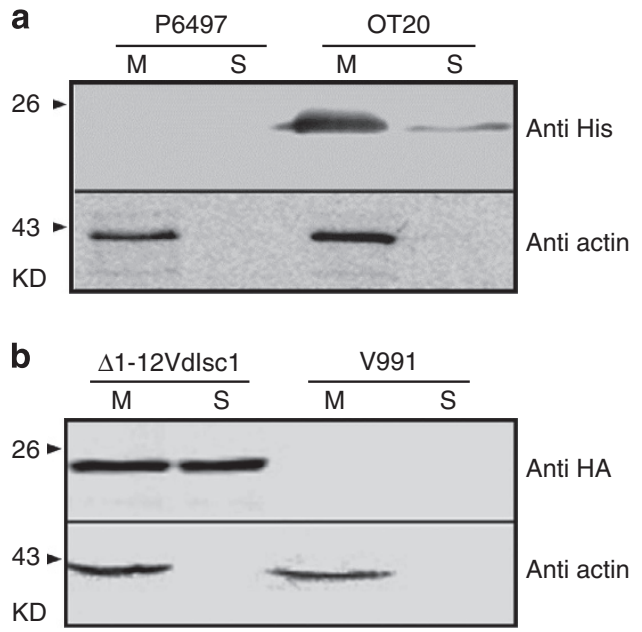

Figure 3 | PsIsc1 and Vdlsc1 are secreted proteins. Pslsc1-His (a) and Vdlsc1-HA (b) were expressed in P. sojae (OT20) and V. dahliae ( $\Delta 1-$ $12 \mathrm{~V} d \mathrm{sc} 1)$, respectively. Proteins extracted from mycelia $(M)$ and culture supernatants $(S)$ were analysed using western blotting with anti-His, -HA or $-\alpha$-actin antibodies, as indicated. Extracts and supernatants from non-transformed P. sojae P6497 and V. dahliae V991 were used as controls for antibody specificity.

PsIsc1 is translocated into host cells during interactions. To assess the localization of PsIsc1 during infection, we generated the $P$. sojae transformants producing the coding region of monomeric red fluorescent protein (mRFP) fused to the $C$ terminus of PsIsc1 or the P. sojae RxLR effector Avr1b ${ }^{19}$. PsIsc1-mRFP fusion protein was validated in both mycelia and culture supernatants, and was the same as the Avr1b-mRFP. In contrast, soluble protein green fluorescent protein (GFP) was detected in mycelia, but not in the culture supernatants (Supplementary Fig. 6). These results further indicate that PsIsc1 and Avrlb are secreted proteins. Avrlb-mRFP was predominantly localized in haustoria and the extrahaustorial matrix during soybean infection. Fluorescence was not detected within infected cells, presumably due to the dilution of Avrlb-mRFP within the plant cells (Fig. 4a), as observed with $P$. infestans Avr3a ${ }^{23}$. PsIsc1-mRFP also showed strong localization in haustoria and the extrahaustorial matrix, but was not detected in plant cells. The unfused control (mRFP) did not show haustorial localization (Fig. 4a). Therefore, PsIsc1 is distributed in a similar manner as the host cytoplasmic effector Avrlb, and both proteins might be secreted from haustoria during interactions between $P$. sojae and soybean.

To determine whether PsIsc1 enters host cells following secretion, we examined whether it could deliver Avrlb into host soybean cells. Oomycete RxLR effectors such as the P. infestans effector Avr3a ${ }^{23}$ and $P$. sojae Avr1b $b^{19,24}$ depend on their N-terminal RxLR-dEER domain for host cell delivery. We demonstrated previously that the transformants expressing WT Avr1b lost the ability to infect soybean plants carrying Rps $1 \mathrm{~b}$ but retained the ability to infect plants lacking $R p s 1 \mathrm{~b}$, and that the $\mathrm{N}$-terminal RxLR-dEER domain was required its translocation into host cells ${ }^{19}$. We deleted the N-terminal region from Avr1b (producing Avr1bCt) and replaced it with PsIsc1 (producing PsIsc1-Avr1bCt), and then generated two P. sojae transformants that expressed the fused PsIsc1-Avr1bCt (Fig. 4b). Production of the expected proteins was confirmed using western blotting (Fig. 4c). Transformants expressing the fused Avrlb-mRFP were consistently avirulent on soybean cultivars carrying Rps $1 \mathrm{~b}$ (Fig. 4d), suggesting that the recognition of Avr1b inside soybean cells was not affected by the fusion with mRFP. The inoculation 
a
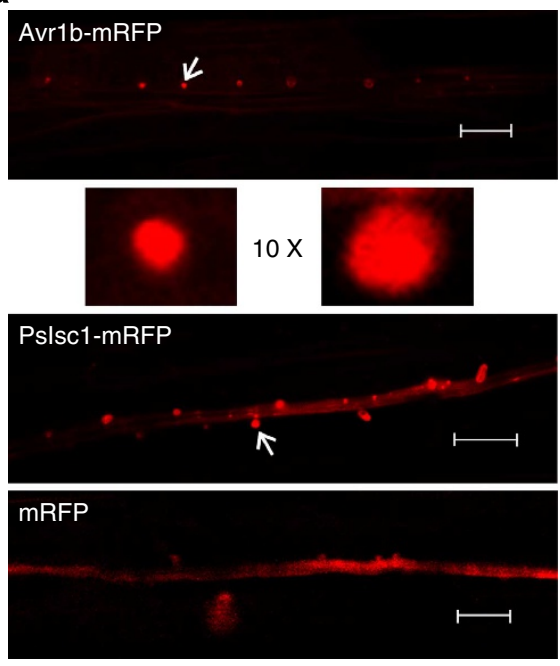

d

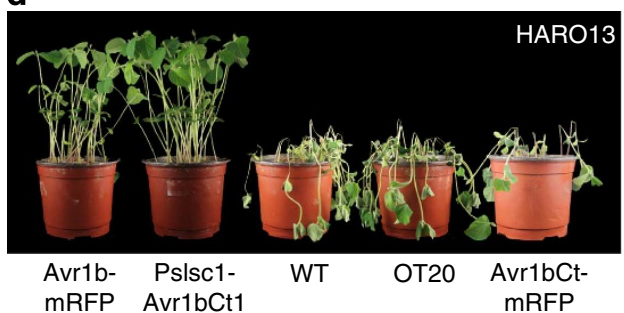

b

$$
\begin{array}{l|l|l|l|}
\text { Avr1b } & \text { SP } & \text { RxLR-dEER } & \text { Avr1bCt } \\
\hline
\end{array}
$$

\begin{tabular}{l|l|l|l|l|l|}
\hline Avr1b-mRFP & SP & RxLR-dEER & Avr1bCt & mRFP & His \\
\hline
\end{tabular}
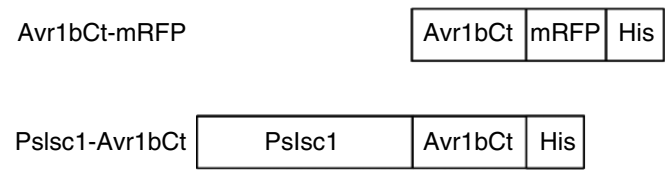

C
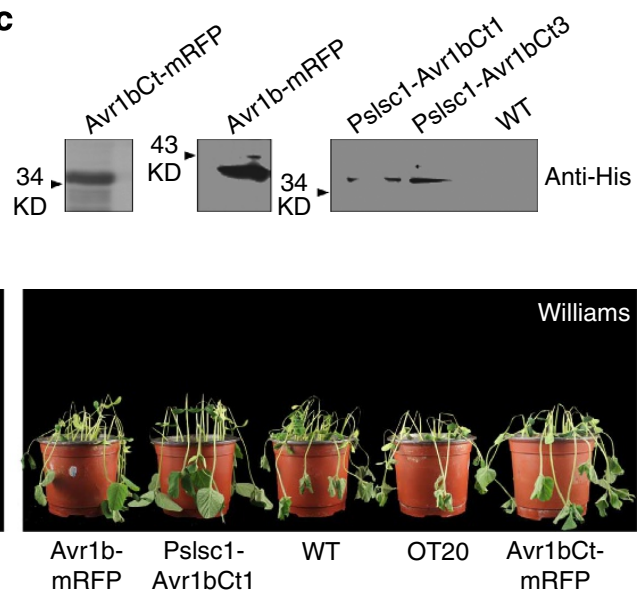

Figure 4 | Pslsc1 can replace the translocation domain of Avr1b. (a) Pslsc1 preferentially accumulates in haustoria. P. sojae-infected hyphae were observed using confocal microscopy at $10 \mathrm{hpi}$. P. sojae expressing Avr1b-mRFP showed specific haustorial accumulation (indicated by the arrow) of fluorescent Avr1b (red, upper panel), whereas Pslsc1-mRFP accumulated preferentially in haustoria (red, middle panel). P6497 expressing mRFP served as a control (lower panel). Scale bars, $20 \mu \mathrm{m}$. The arrow indicated haustoria were shown with tenfold magnification. (b) Structure of the fusion of full-length Pslsc1 and the C terminus of Avr1b (Avr1bCt: aa, 66-138, removing the signal peptide and RxLR-dEER domain). (c) Western blotting of proteins from P. sojae transformants expressing Avr1b-mRFP, Avr1bCt-mRFP and Pslsc1-Avr1bCt (Pslsc1-Avr1bCt1 and Pslsc1-Avr1bCt3). The P. sojae isolate P6497 served as a control for antibody specificity. (d) The phenotypes of hypocotyls from soybean cultivars HARO13 (Rps1b) and Williams (rps). Photographs were taken 2 dpi.

of soybean hypocotyls showed that the two transformants expressing PsIsc1-AvrlbCt were unable to infect the soybean cultivar HARO13 containing the Rps $1 \mathrm{~b}$ resistance gene, but could still infect Williams, which lacks Rps $1 \mathrm{~b}$. In contrast, the recipient WT strain (P6497) and the transgenic lines expressing PsIsc1 (OT20) or Avr1bCt-mRFP could kill both cultivars (Fig. 4d). Similar results were observed using a leaf inoculation assay (Supplementary Fig. 7). Taken together, these data suggest that PsIsc1 could functionally replace the secretory leader and host cell entry domain of Avrlb, and that PsIscl might be translocated inside host cells during infection.

PsIsc1 and VdIsc1 suppress SA-mediated defense in planta. To determine whether PsIsch1 and VdIsch1 function within plant cells to compromise immunity, we expressed these two genes (as GFP fusions) transiently in Nicotiana benthamiana tissue using Agrobacterium infiltration. Western blotting with anti-GFP antibodies confirmed the expression of proteins of the expected length (Fig. 5a). We also assessed the distribution of PsIsc1 and VdIsc1 in plant cells. Neither of the two proteins were co-localized with the mCherry-tagged plastid marker pt-rk CD3-999 (ref. 25), but were instead localized to the cytoplasm and nucleus of transformed N. benthamiana cells, similar to native GFP alone (Supplementary Fig. 6). We then inoculated leaves with a compatible pathogen, $P$. capsici. Compared with leaves expressing GFP alone, the diameters of the lesions caused by $P$. capsici were significantly increased following expression of PsIsc1 and VdIsc1 (Fig. 5b,c). The levels of SA (Fig. 5d) and PR1 mRNA (Fig. 5e) in the inoculated sites were reduced when the two genes were expressed, indicating that PsIscl and VdIsc1 suppressed SA-mediated Phytophthora resistance in N. benthamiana. When mutations were introduced into the catalytic triads of PsIsc1 and VdIsc1, they no longer suppressed resistance to $P$. capsici or reduced $S A$ and $P R 1$ transcript levels when transiently expressed in N. benthamiana (Fig. 5).

PsIscl and VdIsc1 have ISC activity. To examine whether PsIsc1 and VdIscl could catalyse hydrolysis of isochorismate into DDHB, we purified targeted proteins from $N$. benthamiana leaves (Fig. 5a) and used E. coli EntB and V. cholerae VibB that were expressed in E. coli as positive controls. The enzyme activity was determined as described previously by measuring the increase in absorbance at $340 \mathrm{~nm}$ caused by $\mathrm{NAD}^{+}$reduction ${ }^{13}$. In plantaexpressed PsIsc1 and VdIsc1, as well as two positive controls, exhibited high enzyme activity. Mutations in the catalytic triads of PsIsc1 and VdIsc1 resulted in an almost complete loss of enzyme activity. No activity was detected when GFP (a negative control) was purified and analysed using the same procedures (Fig. 5f). We also measured the relative levels of DDHB in plant tissues expressing the above genes. Significantly increased DDHB concentrations were detected in total extracts of the leaves expressing PsIsc1 or VdIsc1, whereas DDHB accumulated at 
a

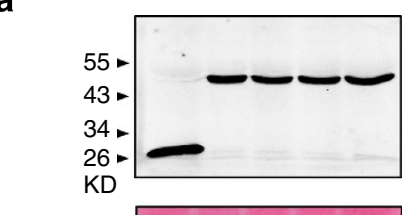

Ponceau S b

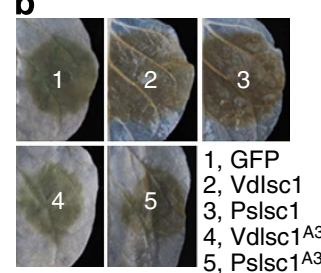

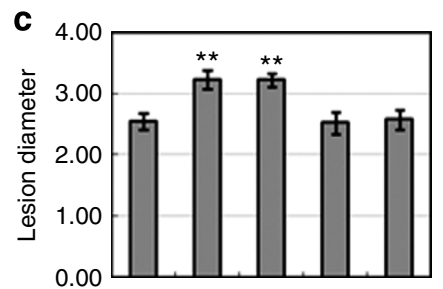
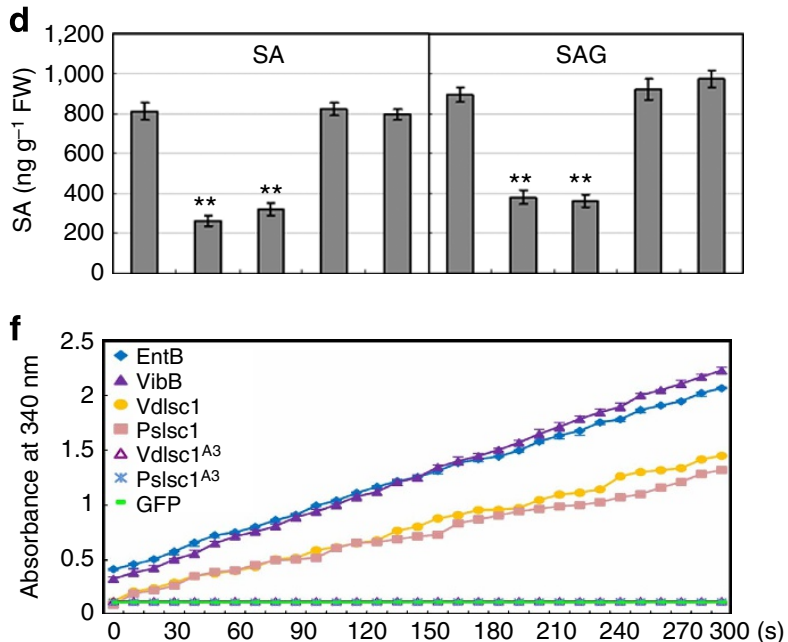
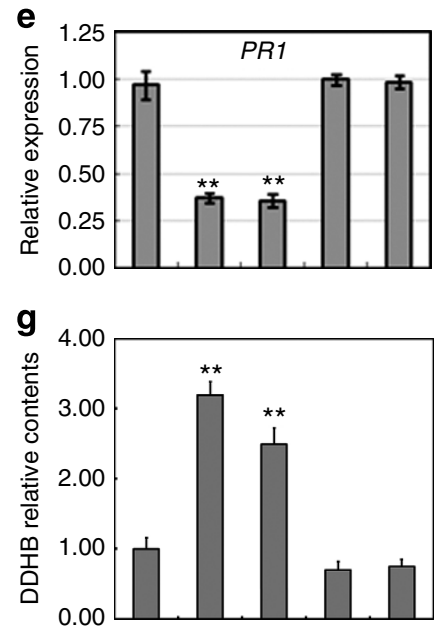

Figure 5 | Pslsc1 and Vdlsc1 suppress disease resistance. (a) Western blotting of the indicated transiently expressed proteins in N. benthamiana using anti-GFP antibodies. (b) Phenotypes of leaves transiently expressing the indicated genes inoculated with $P$. capsici zoospores. Photos were taken at 48 hpi. (c) Lesion diameters of infected regions at 48 hpi, averaged from at least 30 inoculated sites. (d) Free SA (left) and SAG (right) levels (12 hpi) in leaves transiently expressing the indicated genes. (e) Relative levels of PR1 gene transcripts at 12 hpi in infected leaves transiently expressing the indicated genes. (f) Isochorismatase activity by measuring the absorbance at $340 \mathrm{~nm}$. Bacterial EntB and VibB produced by E. coli were used as positive controls. An identical quantity of the tested proteins was purified from plant tissues. VibA generates NADH from DDHB and NAD ${ }^{+}$when active ISC was present (producing DDHB from isochorismate); the absorbance of $\mathrm{NADH}$ at $\mathrm{A} 340 \mathrm{~nm}$ at the indicated time points (seconds) was recorded. (g) Relative DDHB concentrations in plant tissues expressing the indicated genes. The relative DDHB concentration of each tested sample was calculated based on $\mathrm{A} 340$ absorbance and the DDHB concentration in GFP-expressing leaves was normalized to 1.0. In a, c-e and $\mathbf{g}$, from left to right: GFP, Vdlsc1, Pslsc1, Vdlsc1 A3 and Pslsc1 ${ }^{\mathrm{A} 3}$. ${ }^{\star \star} P<0.01$ (Dunnett's test).

relatively lower levels in leaves expressing GFP, $P s I s c 1^{A 3}$ or $V d I s c h 1^{A 3}$ (Fig. 5g). Therefore, both proteins have ISC activity.

$\mathrm{N}$ termini of PsIsc1 and VdIsc1 are required in $V$. dahilae. PsIsc1 and VdIsch1 contain no motifs that are similar to $\operatorname{RxLR}^{19,23}$. We constructed mutants of both proteins lacking the N-terminal regions (PsIsch $1 \Delta^{\mathrm{Nt}}$ and $\mathrm{VdIsch} 1 \Delta^{\mathrm{Nt}}$ ) (Supplementary Fig. 2). In these mutants, the NN scores calculated using the SecretomeP 2.0 server were below the normal threshold for secreted proteins ${ }^{22}$ (Supplementary Fig. 2). Neither of the deletion mutants complemented the phenotypes of VdIsc1-deletion mutants in cotton seedlings (Fig. 6a), suggesting that both proteins require their putative unconventional secretion signals to function in $V$. dahliae. To confirm that loss of the $\mathrm{N}$-terminal regions did not compromise ISC activity, we transiently expressed PsIsc $1 \Delta^{\mathrm{Nt}}$ and $V d I s c 1 \Delta^{\mathrm{Nt}}$ in $N$. benthamiana leaves. The deletion mutants conferred similar phenotypes as their WT in terms of the levels of resistance (Fig. 6b), the SA/SAG content and PR1 transcripts (Fig. 6c). Therefore, the N-terminal region is probably required for the secretion of these proteins from pathogens, but not for their roles in plant cells.

\section{Discussion}

Little is known about how filamentous plant pathogens interfere with host innate immunity for successful colonization and infection. As SA-mediated signalling pathways are important for plant immune responses and isochorismate is a precursor of SA, it has been inferred that phytopathogens could produce ISCs to reduce SA accumulation in response to pathogen attack and thus inhibit plant defense responses ${ }^{16,17,26}$. In the current study, we have demonstrated experimentally that $P$. sojae and $V$. dahliae encode ISC effectors (PsIscl and VdIsc1) to disrupt the SA metabolism pathway. Notably, we provide evidence that the ISCs are probably secreted from the two filamentous pathogens through unconventional pathways, and that PsIscl can enter into host cells during infection.

It was reported that ISC family motifs were found in the secretome of some phytopathogenic filamentous ascomycete fungi, but not in non-phytopathogenic species ${ }^{16}$ Isochorismatase hydrolase was also identified specifically in a highly aggressive isolate of $V$. dahliae using proteomics-based analyses ${ }^{26}$. We identified putative ISC genes in $P$. sojae and $V$. dahliae (PsIsc1 and VdIsc1, respectively) based on primary and secondary structural similarities with known ISCs and demonstrated that both genes were highly expressed during infection stages. The overexpression or silencing of PsIsc1 in $P$. sojae led to increased and reduced virulence, respectively; SA levels in infected host tissues were altered accordingly. Similar results were observed when VdIsc1 was deleted in V. dahliae. Interestingly, the PsIscl gene could functionally replace $V d I s c 1$ in $V$. dahliae. The transient overexpression of these two genes in $N$. benthamiana tissues suppressed SA levels and SA-mediated defense; consistent with this, DDHB levels were increased. Furthermore, we demonstrated that the conserved ISC catalytic 
a

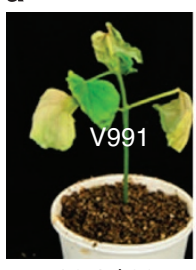

$36.1 / 92$

b

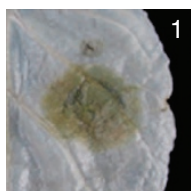

$2.65 \pm 0.07$

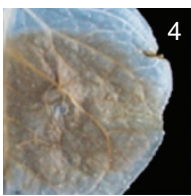

$3.37 \pm 0.08$

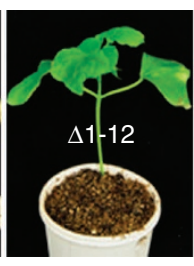

$14.4 / 91$

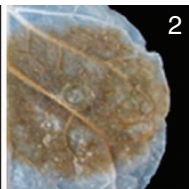

$3.23 \pm 0.07$

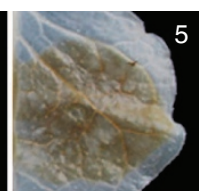

$3.38 \pm 0.06$

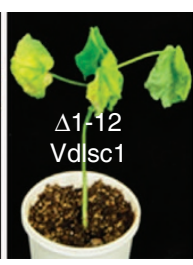

33.4 / 94

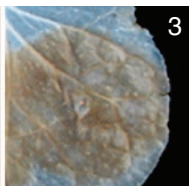

$3.23 \pm 0.09$

1, GFP

2, Vdlsc1

3, Pslsc1

4, Vdlsc ${ }^{\Delta \mathrm{Nt}}$

5, $\mathrm{Pslsc}{ }^{\Delta \mathrm{Nt}}$

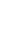

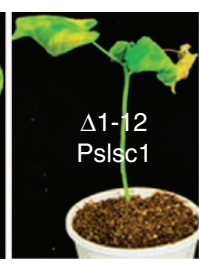

36.8 / 97

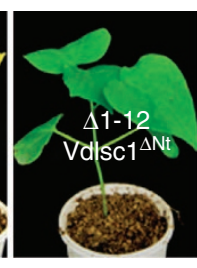

16.8 / 93

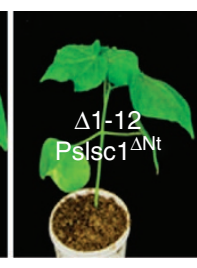

$16.4 / 90$

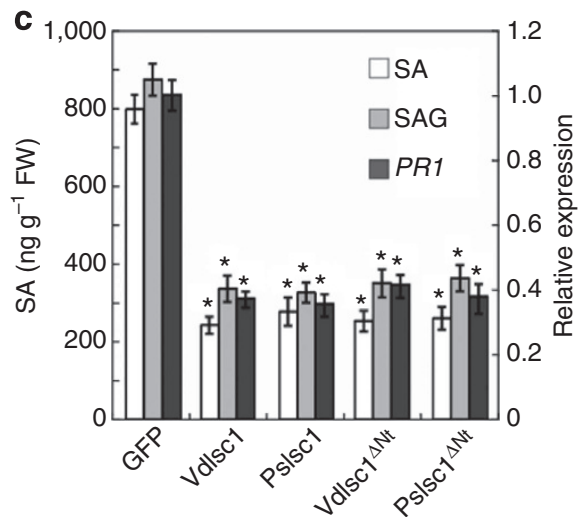

Figure 6 | Functional analysis of the $\mathbf{N}$ termini of Pslsc1 and Vdlsc1. (a) Phenotypes of cotton seedlings inoculated with the indicated strains. The photos were taken at $15 \mathrm{dpi}$ and a typical seedling is shown as an example for each line. The numerator indicates the average disease index,

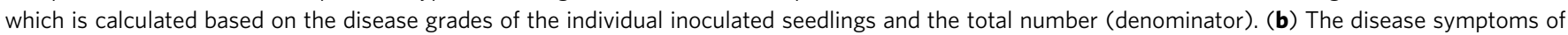
leaves transiently expressing the indicated genes that were inoculated with $P$. capsici zoospores. Photos were taken at 48 hpi. The numbers indicate the lesion diameters of infected regions that were scored at $48 \mathrm{hpi}$ and represent the mean at least 30 inoculated sites. (c) The relative levels of SA, SAG (left axis) and PR1 transcripts (right axis) at $12 \mathrm{hpi}$ in infected leaves transiently expressing the indicated genes. ${ }^{\star} P<0.01$ (Dunnett's test).

triads in PsIsc1 and VdIsc1 were required for ISC activity and the suppression of plant immunity. Thus, we conclude that these two evolutionary distinct pathogens both generate ISCs to promote infection by destroying a precursor (isochorismate) of the plant defense-signalling hormone SA.

As summarized in Fig. 7, several distinct mechanisms have been adopted by phytopathogens to suppress host SA biosynthesis by cell-entering effectors. The Pseudomonas syringae effector HopI1 remodels host chloroplast thylakoid structure and suppresses SA accumulation via an unknown mechanism ${ }^{27}$. Ustilago maydis secretes a host cytoplasmic effector (Cmu1), which is a chorismate mutase that converts chorismate to prephenate. As chorismate is a key intermediate in the biosynthesis of $\mathrm{SA}^{10}, U$. maydis can effectively deplete chorismate and suppress SA accumulation via Cmul (ref. 28). Secreted chorismate mutases are found in many plant fungal and nematode pathogens ${ }^{28,29}$. In the current study, we demonstrated that phytopathogens from two kingdoms have evolved a novel strategy to inhibit SA biosynthesis in plants by producing ISC effectors. It is likely to be that neither of the pathogen-generated ISCs are imported into the plastids of host plants, because neither PsISC1 nor VdISC1 were co-localized with the plastid marker pt-rk CD3-999 (ref. 25) when they were transiently co-expressed in the leaves of N. benthamiana (Supplementary Fig. 8). However, when delivered into host plant cells by the pathogens, the ISC effector proteins might be able to affect the cellular homeostasis of isochorismate. Although SA is thought to be synthesized in the plastids, plastid-produced isochorismate may transport to the cytosol where it acts as the substrate of VdIsc1 or PsIsc1 to be catalysed into DDHB. Reduction of cytosolic concentrations of isochorismate by pathogen-derived ISC effectors in turn may enhance its export from the plastid to the cytosol. A similar mechanism was also proposed to explain how U. maydis CMU1 acts on plant chorismate ${ }^{28}$.

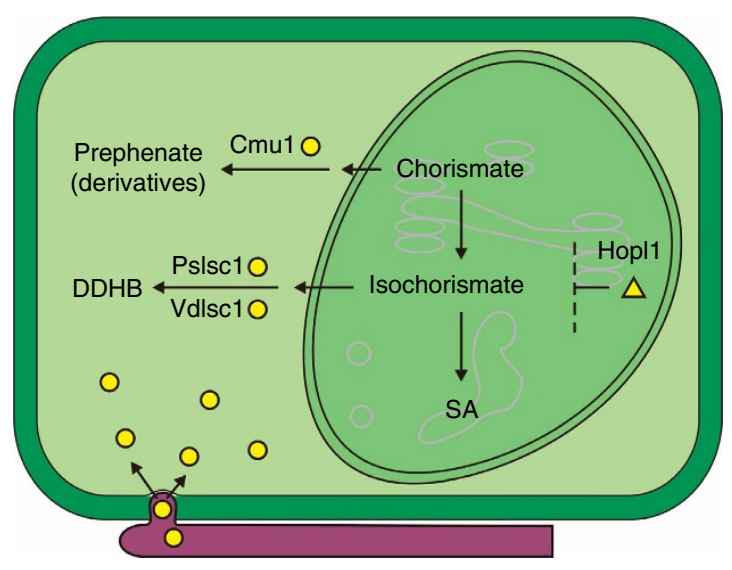

Figure 7 | Pathogen effectors subverting plant SA biosynthesis. Pathogens use cell-entering effectors (yellow) to suppress plant SA biosynthesis in distinct manners. Filamentous pathogen-derived enzymatic effectors (circle) inhibit SA accumulation by redirecting its precursors from plastid (darker green) to cytosol (mint green), whereas bacterial effector Hopl1 (triangle) depresses SA levels by remodelling chloroplast thylakoid structure.

Secreted proteins containing ISC family motifs have been found in several fungal and nematode pathogens ${ }^{16,17}$, suggesting a common strategy for host manipulation. Therefore, different phytopathogens have evolved distinct intracellular effectors independently to actively suppress SA accumulation in host cells. The findings suggest a promising strategy for disease control by inhibiting the activities of these enzymatic effectors or supplying the host with plant-defense activators to compensate for the SA shortage caused by pathogen effectors. For example, 
several highly active inhibitors targeting the secreted chorismate mutase from Mycobacterium tuberculosis have been designed and evaluated based on structure-guided approaches ${ }^{30}$. Our results suggest that secreted ISCs from phytopathogens could be suitable targets for disease control.

We further showed that PsIscl and VdIscl are secreted proteins, although neither protein contained a predicted signal peptide when we analysed using two widely used algorithms ${ }^{31,32}$. Proteins that lack signal peptides can access the cell exterior via the unconventional protein secretion pathway, which has been documented in animal, plant, bacterial and yeast cells ${ }^{33,34}$. Indeed, both proteins were predicted to be unconventionally secreted proteins using an available algorithm ${ }^{22}$. The secretion mode of ISCs suggests that unconventional secretion occurs in $P$. sojae and $V$. dahliae. At least four distinct transport pathways are involved in unconventional secretion ${ }^{34}$, but it remains unknown which (if any) of these mechanisms are used by these two filamentous pathogens. The recruitment of unconventional secretion for effector delivery in both an oomycete and a fungal plant pathogen further broadens the extensive resources pathogens can bring to bear against hosts, and broadens the range of proteins that bioinformaticians must examine as potential effectors.

We used P. sojae to examine the pathway of PsIsc1 secretion and host entry in more detail. We demonstrated that PsIsc1 accumulates predominantly in haustoria and the extrahaustorial matrix during soybean infection, which is a similar distribution to that of well-characterized cell-entering effectors, such as $P$. sojae $A_{\text {Avr } 1 b^{19}}$ and $P$. infestans Avr $3 \mathrm{a}^{23}$. We did not observe the translocation of fluorescent protein fusions of PsIsc1 inside host cells during infection, which might be due to dilution of the fluorescence signal in plant cell cytoplasm. Nevertheless, we demonstrated that PsIscl could functionally replace the $\mathrm{N}$-terminal regions (host target signals, including the signal peptide and RxLR-dEER domain) in Avrlb and mediate the translocation of Avrlb into host cells, consistent with the prediction that PsIscl enters into host cells during infection to metabolize the SA precursor. The observation that PsIsc1 was functional when expressed in $V$. dahliae suggests that either PsIsc1 does not rely on any pathogen-encoded machinery for entry, or that the two pathogens produce functionally analogous translocation machinery.

Assessing how the effectors from filamentous pathogens are translocated into host cells is a major focus of current research. In oomycete pathogen effectors, the RxLR-dEER domain is considered to mediate host cell entry by binding to phosphatidylinositol phosphates on the outer surface of plant plasma membranes $^{24}$. Consistent with this, Phytophthora pathogens could generate external phosphatidylinositol-3-phosphate to promote the entry of RxLR effectors ${ }^{35}$. In contrast, Magnaporthe oryzae intracellular effectors accumulate preferentially in the biotrophic interfacial complex, a novel interfacial structure that is associated with the initial invading hyphae $e^{36,37}$, suggesting that the effectors may be translocated through a specialized structure in this fungal pathogen. In the current study, we showed that both PsIsc1 and VdIscl probably function inside host cells and PsIsc1 accumulates in haustoria. Nevertheless, the exact mechanism behind the translocation is yet to be investigated.

In summary, these results provide evidence that $P$. sojae and $V$. dahliae produce a novel class of effectors: ISC enzymes that destroy the precursor of the plant defense-signalling hormone SA. The discovery of these enzymes in both fungi and oomycetes suggests that this is a central mechanism of virulence in filamentous plant pathogens. We also demonstrated that unconventional secretion has been recruited by plant pathogens in two kingdoms to deliver effectors into host plants; in addition to the biological novelty, this discovery transforms the way that candidate effectors should be identified, which is at present heavily reliant on the identification of signal peptides.

\section{Methods}

Bioinformatic analyses. Known ISC protein sequences were obtained from the PDB database (http://www.rcsb.org) ${ }^{38}$ and were used as queries to search against the $P$. sojae $e^{1}$ and $V$. dahliae $e^{2}$ protein sequence databases using reciprocal Blast searches programmes. All the obtained proteins were analysed using HHpred ${ }^{18}$ against the PDB and PFAM databases (http://pfam.sanger.ac.uk/) ${ }^{39}$. Proteins were considered to be putative ISCs only if they met the following criteria: in a search of the PFAM database, the proteins were classified as a member of the PF00857 family, which groups together ISCs; and the HHpred searches identified the proteins as belonging to the ISC family (the top ten hits contain over five ISCs). The putative ISCs identified were further confirmed using annotations in the NCBI database.

Sequence alignments were generated using the MUSCLE algorithm ${ }^{40}$ Secondary structure was predicted using Porter (http://distill.ucd.ie/porter/) ${ }^{41}$ and then compared with the known crystal structures of ISCs from Oleispira antarctica (PDB code: 3LQY) ${ }^{20}$, Leishmania major (PDB code: 1XN4) and Trypanosoma cruzi (PDB code: $1 Y Z V)^{15}$. Signal peptide prediction was performed using SignalP 4.1 (http://www.cbs.dtu.dk/services/SignalP/) ${ }^{32}$ and iPSORT (http://ipsort.hgc.jp/) ${ }^{31}$. Non-classically secreted proteins were predicted using SecretomeP 2.0 (http:// www.cbs.dtu.dk/services/SecretomeP/) with the threshold of neural network output score of 0.5 as recommended by the algorithm ${ }^{22}$.

Microbial strains and plant lines. All of the pathogen strains, the derived transgenic $P$. sojae and $V$. dahliae lines, and plant materials used in the current study are summarized in Supplementary Table S3. P. sojae and $P$. capsici strains were grown and maintained as described previously ${ }^{35}$. V. dahliae strain V991, a highly toxic defoliating WT strain, was grown on PDA medium at $25^{\circ} \mathrm{C}^{42}$. E. coli and Agrobacterium tumefaciens were grown using appropriate selectable markers. All plants were grown in growth chambers at appropriate temperatures with 16-h photoperiods and $55 \%$ humidity.

Generation of transformants and virulence assays. Transformed $P$. sojae lines were obtained using polyethylene glycol-mediated protoplast transformation procedure ${ }^{19,35}$. Putative transformants were screened by quantitative reverse transcriptase (qRT)-PCR to identify silenced or overexpression transgenic lines. The overexpression lines were confirmed by western blotting using antibodies against the relevant tags. The pathogenicity phenotypes of selected $P$. sojae transformants were determined by hypocotyl inoculation of etiolated soybean seedlings. Approximately 100 zoospores of each transformant and the WT strain were inoculated onto the hypocotyl of etiolated (susceptible Williams) soybean seedlings. The avirulence phenotypes of selected transformants were evaluated using hypocotyl inoculation ${ }^{19}$ with the soybean cultivars Williams (rps) and HARO13 (Harosoy background, Rps1b). Each avirulence or virulence determination was repeated at least three times. Significant differences were identified using Dunnett's test.

$V$. dahliae transformation was performed using Agrobacterium tumefaciensmediated transformation ${ }^{43}$. Briefly, the conidia $\left(1 \times 10^{8}\right.$ spores per $\left.\mathrm{ml}\right)$ were harvested and then mixed with an equal volume of $A$. tumefaciens containing the appropriate vector and $200 \mu \mathrm{M}$ acetosyringone. After incubation, the cultures were transferred to PDA medium containing $50 \mu \mathrm{gl}^{-1}$ hygromycin B (Roche, Switzerland). The putative transformants were screened for gene deletions or the presence of transgenes using genomic PCR and qRT-PCR. For complementation, the mutant strains were further transformed with VdIsc1, PsIscl and the different mutants fused with an HA tag. For cotton and Arabidopsis infection assays, plants were inoculated with $V$. dahliae WT or transformants by conidial root dip ${ }^{42}$. For Arabidopsis inoculations, we modified the dip time to $5 \mathrm{~min}$ and the inoculum to $1 \times 10^{7}$ conidia per $\mathrm{ml}$. The symptoms were evaluated and the disease grade was classified as follows: 0 (no symptoms), 1 ( $>0-25 \%$ wilted leaves), 2 (25-50\%), 3 $(50-75 \%)$ and $4(75-100 \%)$. The data were analysed using Student's $t$-test.

Molecular experiments. Promoters, flanking sequences, complete open reading frames and/or fragments of PsIsc1, VdIsc1, Avrlb and EntC were amplified by PCR using specific primers (Supplementary Table 4) and cloned via standard restriction digest and ligation methods to produce expression constructs based on the vectors pC1300, pTOR, pET21b, pBinGFP2 or pBinGFP4 (Supplementary Table 5).

Total RNA was extracted from cotton roots using the CTAB method ${ }^{42}$. Total RNA was extracted from $N$. benthamiana leaves, etiolated soybean seedlings, and $P$. sojae and $V$. dahliae mycelium using RNA simple Total RNA Kit (Tiangen, China) following the manufacturer's instructions. qRT-PCR was performed using SYBR green real-time RT-PCR assays (TaKaRa) as described previously ${ }^{35}$. To assess the transcriptional profile of genes from $P$. sojae, total RNA was collected from $P$. sojae P6497 mycelium sandwiched between pairs of soybean leaves. The mycelium was harvested at 2, 4 and $10 \mathrm{~h}$ after inoculation (hpi) and the infected soybean leaves, including mycelia, were collected at 12, 24, 36 and 48 hpi. To assess 
the transcriptional profile of genes from $V$. dahliae inoculum, mycelia were inserted into wounded $N$. benthamiana petioles and the mycelia were recovered at $6,12,24$ hpi, and the infected petioles, including mycelia, were also collected at 3 and 5 dpi.

Western blotting. Protein extracts were prepared by grinding $\sim 100 \mathrm{mg}$ leaf or slightly dried mycelia samples in liquid nitrogen. The resulting powder was then suspended in protein extraction buffer $(50 \mathrm{mM}$ HEPES, $150 \mathrm{mM} \mathrm{KCL}, 1 \mathrm{mM}$ EDTA, $0.1 \%$ Triton X-100, pH 7.5) supplemented with $1 \mathrm{mM}$ dithiothreitol and $1 \times$ protease inhibitor cocktail (Roche). The resultant suspensions were mixed and centrifuged at $12,000 \mathrm{~g}$ at $4{ }^{\circ} \mathrm{C}$ for $15 \mathrm{~min}$ and the supernatants were recovered. Samples were fractionated using standard SDS-PAGE gels (12\% acrylamide) and blotted using standard procedures ${ }^{44}$. Anti-His-tag primary monoclonal antibody (Sigma-Aldrich) and IRDye 800CW-conjugated goat (polyclonal) anti-mouse IgG $(\mathrm{H}+\mathrm{L}$; LI-COR Biosciences) secondary antibodies were used to assess P. sojae transformants. Anti-GFP monoclonal antibody (Sigma-Aldrich) and IRDye $800 \mathrm{CW}$-conjugated goat anti-mouse IgG secondary antibodies were used to assess transient gene expression in plants. All the primary antibodies were diluted with $5 \%(\mathrm{~m} / \mathrm{V})$ non-fat dried milk in PBS $(1: 3,000)$ and the secondary antibodies were diluted with $5 \%(\mathrm{~m} / \mathrm{V})$ non-fat dried milk in PBS $(1: 10,000)$. The membranes were then visualized using a LI-COR Odyssey scanner with excitation at 700 and $800 \mathrm{~nm}$. The molecular marker is shown in Supplementary Fig. 6 and blots were shown with at least one size-labelled marker.

$V$. dahliae strain $\Delta 1-12 \mathrm{VdIsc1}$, P. sojae strains OT20, PsIsc1-mRFP, and Avr1bmRFP and GFP were used to analyse the secretion of VdIscl and PsIscl. The proteins were purified from the same amounts of culture (including the mycelia and supernatants) for each strain. For $P$. sojae, mycelia were cultivated in flasks at $25^{\circ} \mathrm{C}$ with shaking at 100 r.p.m. for 2 weeks in liquid Czapek culture medium. Culture supernatants were precipitated using ammonium sulphate and then analysed using western blotting with mouse anti-His (Sigma-Aldrich) and anti- $\alpha$ actin antibodies (Sigma, A3853). For $V$. dahliae, mycelia were cultivated in a flask for 2 weeks at $25^{\circ} \mathrm{C}$ in liquid Czapek-Dox medium and the secreted proteins were collected following the reported procedures ${ }^{45}$. Culture supernatants were precipitated using trichloroacetic acid and then analysed using western blotting with mouse-anti HA (Sigma, H3663) and anti- $\alpha$-actin antibodies.

N. benthamiana infiltration and virulence assays. A. tumefaciens strain GV3101 containing the appropriate vector was infiltrated into N. benthamiana leaves ${ }^{35}$. $P$. capsici infection assays were performed using droplet inoculations of zoospore solutions of $P$. capsici isolate 35 (10 $\mu \mathrm{l}, \sim 500$ zoospores) onto detached $N$. benthamiana leaves. At least ten independent (4-week-old) $N$. benthamiana leaves were tested per construct combination. To explore the effect of transient expression on infection, proteins were first expressed by infiltrating cells of the relevant $A$. tumefaciens strain into $N$. benthamiana leaves $24 \mathrm{~h}$ before $P$. capsici inoculation. The growth efficiency of $P$. capsici was quantified by measuring the lesion diameter $(\mathrm{mm})$ at $48 \mathrm{hpi}$.

Measurement of SA/SAG concentrations and application. SA was extracted from leaves excised from soybean, cotton and N. benthamiana plants, and quantified using HPLC as described ${ }^{46}$ with minor modifications. Briefly, leaf powder $(\sim 1.0 \mathrm{~g})$ was ground in liquid nitrogen and then suspended in $90 \%(\mathrm{v} / \mathrm{v})$ methanol. As an internal standard for SA, $100 \mu \mathrm{g}$ 3-hydroxy benzoic acid in $100 \%$ methanol were added to each sample. The SA solution was filtered and separated on a C18 analytical column using HPLC and detected using fluorescence (excitation at $305 \mathrm{~nm}$, emission at $405 \mathrm{~nm}$; Waters). The HPLC was programmed for isocratic conditions with a flow rate of $0.5 \mathrm{ml} \mathrm{min}^{-1}$. To assay SAG, the aqueous phase containing SAG was acidified to $\mathrm{pH} 1.0$ using $\mathrm{HCl}$ and was then boiled for $30 \mathrm{~min}$ to release SA from any acid-labile conjugated forms. The released SA was extracted using an organic mixture and treated as described above. SA and SAG were quantified by area integration of the HPLC peaks.

Cotton plants were sprayed with $2.0 \mathrm{mM}$ SA 1 day before inoculation or were mock treated with water before infection. Following inoculation, the plants were sprayed with SA every 7 dpi.

Enzyme activity assays and measurement of DDHB content. Isochorismatase activity was measured as described previously ${ }^{13}$. Briefly, the enzyme EntC was used to convert chorismate to isochorismate and enzyme activity was then determined by measuring the increase in absorbance at $340 \mathrm{~nm}$ caused by the production of $\mathrm{NADH}$ from $\mathrm{NAD}^{+}$when $V$. cholerae VibA was present in the reaction solution (converting $\mathrm{DDHB}+\mathrm{NAD}^{+}$to 2,3-DHB $+\mathrm{NADH}$ ) using an Uvikon spectrophotometer. Purified E. coli EntB and V. cholerae VibB produced from E. coli were used as positive controls ${ }^{13}$. The tested proteins were purified from N. benthamiana transiently expressing VdIscl or PsIscl genes using HisTrap HP (GE Healthcare) following the manufacturer's instructions. The assay was performed in a total volume of $100 \mu \mathrm{l}$ containing $10-\mu \mathrm{l}$ reaction solutions from the first step, $100 \mathrm{mM}$ PBS buffer $\mathrm{pH} 7.0,0.8 \mathrm{mM} \mathrm{NAD}^{+}, 4 \mu \mathrm{g}$ of each purified protein and $3.8 \mu \mathrm{g}$ of VibA at room temperature.

To measure the relative DDHB concentration in plant tissues, the crude extracts were collected from $\sim 1 \mathrm{~g} \mathrm{~N}$. benthamiana leaves. The assay was performed in a total volume of $100 \mu \mathrm{l}$ containing $100 \mathrm{mM}$ PBS, $\mathrm{pH} 7.0,10$ - $\mu \mathrm{l}$ crude extracts, $3.8 \mu \mathrm{g}$ purified VibA, $0.8 \mathrm{mM} \mathrm{NAD}^{+}, 100 \mathrm{mM}$ PBS buffer $\mathrm{pH} 7.0$ at room temperature for $10 \mathrm{~min}$. Next, the absorbance at $340 \mathrm{~nm}$ owing to $\mathrm{NAD}^{+}$reduction was used to calculate the relative DDHB contents in the tested samples.

Microscopy. One hundred zoospores from each $P$. sojae transformant were inoculated onto the hypocotyl of the susceptible soybean Williams etiolated seedlings. The surface layer of the inoculated tissue was then examined $\sim 10 \mathrm{hpi}$ GFP-VdIscl and GFP-PsIscl fusions were expressed together with the mCherrylabelled plastid localization marker (pt-rk CD3-999) ${ }^{25}$ in N. benthamiana leaves. Protein fluorescence in the $N$. benthamiana leaves was observed using a ZEISS LSM 710 confocal microscope (Zeiss Microsystems) with a $\times 40$ objective lens (Zeiss) at the specific excitation and emission wavelengths (RFP, 561 and 600-650 nm; GFP, 488 and $495-530 \mathrm{~nm}$; mCherry, 587 and $600-650 \mathrm{~nm}$ ).

\section{References}

1. Tyler, B. M. et al. Phytophthora genome sequences uncover evolutionary origins and mechanisms of pathogenesis. Science 313, 1261-1266 (2006).

2. Klosterman, S. J. et al. Comparative genomics yields insights into niche adaptation of plant vascular wilt pathogens. PLoS Pathog. 7, e1002137 (2011).

3. Kamoun, S. A catalogue of the effector secretome of plant pathogenic oomycetes. Annu. Rev. Phytopathol. 44, 41-60 (2006).

4. Richards, T. A. et al. Evolution of filamentous plant pathogens: gene exchange across eukaryotic kingdoms. Curr. Biol. 16, 1857-1864 (2006).

5. Jones, J. D. \& Dangl, J. L. The plant immune system. Nature 444, 323-329 (2006).

6. Dou, D. \& Zhou, J. M. Phytopathogen effectors subverting host immunity: different foes, similar battleground. Cell. Host. Microbe 12, 484-495 (2012)

7. de Jonge, R. et al. Tomato immune receptor Vel recognizes effector of multiple fungal pathogens uncovered by genome and RNA sequencing. Proc. Natl Acad. Sci. USA 109, 5110-5115 (2012).

8. Dempsey, D. A. et al. Salicylic acid biosynthesis and metabolism. Arabidopsis Book 9, e0156 (2011).

9. Fu, Z. Q. \& Dong, X. Systemic acquired resistance: turning local infection into global defense. Annu. Rev. Plant Biol. 64, 839-863 (2013).

10. Strawn, M. A. et al. Arabidopsis isochorismate synthase functional in pathogeninduced salicylate biosynthesis exhibits properties consistent with a role in diverse stress responses. J. Biol. Chem. 282, 5919-5933 (2007).

11. Serino, L. et al. Structural genes for salicylate biosynthesis from chorismate in Pseudomonas aeruginosa. Mol. Gen. Genet. 249, 217-228 (1995).

12. Drake, E. J., Nicolai, D. A. \& Gulick, A. M. Structure of the EntB multidomain nonribosomal peptide synthetase and functional analysis of its interaction with the EntE adenylation domain. Chem. Biol. 13, 409-419 (2006).

13. Liu, S. et al. Structural insight into the ISC domain of VibB from Vibrio cholerae at atomic resolution: a snapshot just before the enzymatic reaction. Acta Crystallogr. D. Biol. Crystallogr. 68, 1329-1338 (2012).

14. Parsons, J. F. et al. Structure and mechanism of Pseudomonas aeruginosa PhzD, an isochorismatase from the phenazine biosynthetic pathway. Biochemistry 42, 5684-5693 (2003).

15. Caruthers, J. et al. Crystal structures and proposed structural/functional classification of three protozoan proteins from the isochorismatase superfamily. Protein Sci. 14, 2887-2894 (2005).

16. Soanes, D. M. et al. Comparative genome analysis of filamentous fungi reveals gene family expansions associated with fungal pathogenesis. PLoS One 3, e2300 (2008).

17. Bauters, L. et al. Analysis of the transcriptome of Hirschmanniella oryzae to explore potential survival strategies and host-nematode interactions. Mol. Plant Pathol. 15, 352-363 (2013)

18. Soding, J. Protein homology detection by HMM-HMM comparison. Bioinformatics 21, 951-960 (2005).

19. Dou, D. et al. RXLR-mediated entry of Phytophthora sojae effector Avrlb into soybean cells does not require pathogen-encoded machinery. Plant Cell 20, 1930-1947 (2008).

20. Goral, A. M. et al. Crystal structure of a putative isochorismatase hydrolase from Oleispira antarctica. J. Struct. Funct. Genomics 13, 27-36 (2012).

21. Delaney, T. P. et al. A central role of salicylic acid in plant disease resistance. Science 266, 1247-1250 (1994).

22. Bendtsen, J. D. et al. Feature-based prediction of non-classical and leaderless protein secretion. Protein Eng. Des. Sel. 17, 349-356 (2004).

23. Whisson, S. C. et al. A translocation signal for delivery of oomycete effector proteins into host plant cells. Nature 450, 115-118 (2007).

24. Kale, S. D. et al. External lipid PI3P mediates entry of eukaryotic pathogen effectors into plant and animal host cells. Cell 142, 284-295 (2010)

25. Nelson, B. K., Cai, X. \& Nebenfuhr, A. A multicolored set of in vivo organelle markers for co-localization studies in Arabidopsis and other plants. Plant J. 51, 1126-1136 (2007).

26. El-Bebany, A. F., Rampitsch, C. \& Daayf, F. Proteomic analysis of the phytopathogenic soilborne fungus Verticillium dahliae reveals differential protein expression in isolates that differ in aggressiveness. Proteomics 10, 289-303 (2010). 
27. Jelenska, J. et al. A J domain virulence effector of Pseudomonas syringae remodels host chloroplasts and suppresses defenses. Curr. Biol. 17, 499-508 (2007).

28. Djamei, A. et al. Metabolic priming by a secreted fungal effector. Nature 478, 395-398 (2011).

29. Bekal, S., Niblack, T. L. \& Lambert, K. N. A chorismate mutase from the soybean cyst nematode Heterodera glycines shows polymorphisms that correlate with virulence. Mol. Plant Microbe Interact. 16, 439-446 (2003).

30. Munack, S. et al. When inhibitors do not inhibit: critical evaluation of rational drug design targeting chorismate mutase from Mycobacterium tuberculosis. Chem. Biodivers. 9, 2507-2527 (2012).

31. Bannai, H. et al. Extensive feature detection of $\mathrm{N}$-terminal protein sorting signals. Bioinformatics 18, 298-305 (2002).

32. Petersen, T. N. et al. SignalP 4.0: discriminating signal peptides from transmembrane regions. Nat. Methods 8, 785-786 (2011).

33. Ding, Y. et al. Unconventional protein secretion. Trends Plant Sci. 17, 606-615 (2012).

34. Nickel, W. Pathways of unconventional protein secretion. Curr. Opin. Biotechnol. 21, 621-626 (2010).

35. Lu, S. et al. Intracellular and extracellular phosphatidylinositol 3-phosphate produced by Phytophthora species is important for infection. Mol. Plant 6, 1592-1604 (2013).

36. Giraldo, M. C. et al. Two distinct secretion systems facilitate tissue invasion by the rice blast fungus Magnaporthe oryzae. Nat. Commun. 4, 1996 (2013).

37. Khang, C. H. et al. Translocation of Magnaporthe oryzae effectors into rice cells and their subsequent cell-to-cell movement. Plant Cell 22, 1388-1403 (2010).

38. Berman, H. M. The Protein Data Bank: a historical perspective. Acta Crystallogr. A 64, 88-95 (2008).

39. Punta, M. et al. The Pfam protein families database. Nucleic Acids Res. 40, 290-301 (2012).

40. Edgar, R. C. MUSCLE: multiple sequence alignment with high accuracy and high throughput. Nucleic Acids Res. 32, 1792-1797 (2004).

41. Nakai, K. \& Horton, P. PSORT: a program for detecting sorting signals in proteins and predicting their subcellular localization. Trends Biochem. Sci. 24, 34-36 (1999).

42. Zhang, B. et al. Island cotton Gbvel gene encoding a receptor-like protein confers resistance to both defoliating and non-defoliating isolates of Verticillium dahliae. PLoS One 7, e51091 (2012).

43. Mullins, E. D. et al. Agrobacterium-mediated transformation of Fusarium oxysporum: an efficient tool for insertional mutagenesis and gene transfer. Phytopathology 91, 173-180 (2001).
44. Yu, X. et al. The RxLR effector Avh241 from Phytophthora sojae requires plasma membrane localization to induce plant cell death. New Phytol. 196, 247-260 (2012).

45. Medina, M. L. \& Francisco, W. A. Isolation and enrichment of secreted proteins from filamentous fungi. Methods Mol. Biol. 425, 275-285 (2008).

46. Lee, D. H., Choi, H. W. \& Hwang, B. K. The pepper E3 ubiquitin ligase RING1 gene, CaRING1, is required for cell death and the salicylic acid-dependent defense response. Plant Physiol. 156, 2011-2025 (2011).

\section{Acknowledgements}

This work is supported by NSFC (31171831), Natural Science Foundation of Jiangsu Province (BK2012027) and Fundamental Research Funds for the Central Universities (KYTZ201403). We thank Professor Brett Tyler (Oregon State University) and Dr Xiaofeng Cui (Shanghai Institute of Plant Physiology and Shanghai Institute of Entomology, CAS) for useful suggestions and editing of the manuscript.

\section{Author contributions}

T.L., T.S., H.B., L.S., W.L., J.X., S.L., L.C., T.C. and M.Z. performed the wet bench experiments. All authors contributed to experiment design and data analysis. D.D., T.L., T.S. and X.Z. wrote the manuscript with input from all authors. D.D. directed the project.

\section{Additional information}

Supplementary Information accompanies this paper at http://www.nature.com/ naturecommunications

Competing financial interests: The authors declare no competing financial interests.

Reprints and permission information is available online at http://npg.nature.com/ reprintsandpermissions/

How to cite this article: Liu, T. et al. Unconventionally secreted effectors of two filamentous pathogens target plant salicylate biosynthesis. Nat. Commun. 5:4686 doi: $10.1038 /$ ncomms5686 (2014).

(c) (i) This work is licensed under a Creative Commons Attribution 4.0 International License. The images or other third party material in this article are included in the article's Creative Commons license, unless indicated otherwise in the credit line; if the material is not included under the Creative Commons license, users will need to obtain permission from the license holder to reproduce the material. To view a copy of this license, visit http://creativecommons.org/licenses/by/4.0/ 